\title{
Commentary: Focus on the Gut-Kidney Axis in Health and Disease
}

\author{
Yulu Li ${ }^{1}$, Bin Zhu ${ }^{2}$, Chun Yang ${ }^{3}$ and Liying Miao ${ }^{1 *}$ \\ ${ }^{1}$ Department of Nephrology, The Third Affiliated Hospital of Soochow University, Changzhou, China, ${ }^{2}$ Department of Critical \\ Care Medicine, The Third Affiliated Hospital of Soochow University, Changzhou, China, ${ }^{3}$ Department of Anesthesiology and \\ Perioperative Medicine, The First Affiliated Hospital of Nanjing Medical University, Nanjing, China
}

Keywords: chronic kidney diseases, gut microbiota, gut-kidney axis, hemodialysis, bacterial translation

\section{A Commentary on}

Focus on the Gut-Kidney Axis in Health and Disease

by Stavropoulou, E., Kantartzi, K., Tsigalou, C., Konstantinidis, T., Romanidou, G., Voidarou, C., and Bezirtzoglou, E. (2021). Front. Med. 7:620102. doi: 10.3389/fmed.2020.620102

We read with great interest the article by Stavropoulou et al. (1), in which the authors aimed to investigate the interactions between human gut microbiome and kidney diseases. These findings showed that intestinal dysbiosis leads to microbiota shifts, including metabolic disarrangements, inflammation, immunosuppression, and accumulation of uremic toxins, finally resulting in kidney failure.

Accumulating evidence has demonstrated that a bidirectional relationship existed in the host

OPEN ACCESS

Edited by:

Ying-Yong Zhao,

Northwest University, China

Reviewed by:

Samy Hakroush,

University of Göttingen, Germany

${ }^{*}$ Correspondence:

Liying Miao

lymiao@sina.com

Specialty section:

This article was submitted to Nephrology,

a section of the journal

Frontiers in Medicine

Received: 19 February 2021 Accepted: 06 April 2021 Published: 03 May 2021

Citation:

Li Y, Zhu B, Yang C and Miao L (2021)

Commentary: Focus on the

Gut-Kidney Axis in Health and Disease. Front. Med. 8:669561.

doi: 10.3389/fmed.2021.669561 and gut microbiome in various kidney diseases. In this study, there are two issues that should be emphasized: Firstly, we totally agree with the important role of the microbiome in kidney diseases. Actually, as compared with intestinal dysbiosis, the imbalance between intestinal dysbiosis and liver is more important to renal function. The gastrointestinal tract mainly interacts with liver through the portal circulation (2). In enterohepatic circulation, chemicals that enter the digestive tract should be assimilated into portal venous blood by enterocytes, removed from blood by uptake into hepatocytes and secreted into the bile. Then these chemicals are deposited back into the intestinal lumen, which may be reabsorbed and reused by intestinal cells (3). As the intestine barrier is damaged, the liver is exposed to numerous toxic molecules and intestinal bacteria, thereby activating the hepatic innate immune system (4). The disruption of enterohepatic circulation results in the increase of toxins and renal injury. In addition, hepatorenal syndrome (HRS) can be caused by the imbalance between liver and kidney, which affects the kidney. Therefore, the role of hepatic function in gut-kidney axis should not be ignored.

The interaction between the intestinal function and renal function is bidirectional. Kidney diseases also affect the structure of gut microbiota and contributes to dysbiosis. As a result of gastrointestinal disorders associated with dysbiosis, the translocation of microbial compounds have been shown to have proinflammatory and nephrotoxic properties (5). In patients with chronic kidney disease (CKD), dysbiosis may lead to increased uremic toxin levels and then result in CKD progression (6). Hemodialysis is an effective treatment strategy for CKD. Hemodialysis cause the rupture of red blood cells, and then also transiently causes volume deficiency in the human body. In addition, strict control of the volume for patients with hemodialysis will cause loss of blood volume. Then it leads to intestinal hypoperfusion, mucosal ischemia and bacterial translocation (7). Intestinal inflammation and dysfunctional epithelial barrier accelerate systemic translocation of the bacterial-derived uremic toxins, thereby causing oxidative stress damage to the kidney (8). 
In conclusion, we need to pay more attentions to the role of liver in the gut-kidney axis, and we should also focus on the effect of CKD and the changes of volume on gut-kidney axis during the hemodialysis. Further studies are greatly required.

\section{REFERENCES}

1. Stavropoulou E, Kantartzi K, Tsigalou C, Konstantinidis T, Romanidou G, Voidarou C, et al. Focus on the gut-kidney axis in health and disease. Front Med. (2021) 7:620102. doi: 10.3389/fmed.2020.620102

2. Milosevic I, Vujovic A, Barac A, Djelic M, Korac M, Radovanovic Spurnic $\mathrm{A}$, et al. Gut-liver axis, gut microbiota, and its modulation in the management of liver diseases: a review of the literature. Int J Mol Sci. (2019) 20:395. doi: 10.3390/ijms20020395

3. Roberts MS, Magnusson BM, Burczynski FJ, Weiss M. Enterohepatic circulation: physiological, pharmacokinetic and clinical implications. Clin Pharmacokinet. (2002) 41:751-90. doi: 10.2165/00003088-20024110000005

4. Raj D, Tomar B, Lahiri A, Mulay SR. The gut-liver-kidney axis: novel regulator of fatty liver associated chronic kidney disease. Pharmacol Res. (2020) 152:104617. doi: 10.1016/j.phrs.2019.104617

5. Lehto M, Groop PH. The gut-kidney axis: putative interconnections between gastrointestinal and renal disorders. Front Endocrinol. (2018) 9:553. doi: $10.3389 /$ fendo.2018.00553

\section{AUTHOR CONTRIBUTIONS}

All the authors conceived the idea and wrote the manuscript.

6. Al Khodor S, Shatat IF. Gut microbiome and kidney disease: a bidirectional relationship. Pediatr Nephrol. (2017) 32:92131. doi: 10.1007/s00467-016-3392-7

7. Berg RD. Bacterial translocation from the gastrointestinal tract. Adv Exp Med Biol. (1999) 473:11-30. doi: 10.1007/978-1-4615-4143-1_2

8. Chen YY, Chen DQ, Chen L, Liu JR, Vaziri ND, Guo Y, et al. Microbiomemetabolome reveals the contribution of gut-kidney axis on kidney disease. $J$ Transl Med. (2019) 17:5. doi: 10.1186/s12967-018-1756-4

Conflict of Interest: The authors declare that the research was conducted in the absence of any commercial or financial relationships that could be construed as a potential conflict of interest.

Copyright $\odot 2021 \mathrm{Li}$, Zhu, Yang and Miao. This is an open-access article distributed under the terms of the Creative Commons Attribution License (CC BY). The use, distribution or reproduction in other forums is permitted, provided the original author(s) and the copyright owner(s) are credited and that the original publication in this journal is cited, in accordance with accepted academic practice. No use, distribution or reproduction is permitted which does not comply with these terms. 